\title{
DNA vaccine targeting the ectodomain of influenza M2 protein to endolysosome pathway enhances anti-M2e protective antibody response in mice
}

\author{
Jaroslav Hollý, Karolína Tomčíková, Mária Vozárová, Margaréta Fogelová, Lucia Jakubcová, \\ Eva Varečková, František Kostolanský*
}

\begin{abstract}
Institute of Virology, Biomedical Research Center of the Slovak Academy of Sciences, Dúbravská cesta 9, 84505 Bratislava, Slovak Republic
\end{abstract}

Received November 20, 2020; accepted December 12, 2020

\begin{abstract}
Summary.-A promising candidate for developing the universal influenza vaccine is the ectodomain of the M2 protein (M2e). We designed and prepared an experimental DNA vaccine with an improved potential to induce anti-M2e immune response. The sequence for truncated NS1 protein followed by 4xM2e was inserted into the expression vector pTriEx-4 (pEx). M2e repeats were fused to the transmembrane domain and cytoplasmic tail of lysosome-associated membrane glycoprotein 2 isoform A (LAMP-2a) to target the M2e to the endo-lysosome pathway, facilitating increased antigen presentation by MHC II. Using confocal microscope immunofluorescence analysis, we confirmed a strong colocalization of pEx 4M2e-LAMP-2a with early endosomes and a weaker colocalization with late endosomes. BALB/c mice immunized with three doses of pEx 4M2e-LAMP-2a DNA vaccine and challenged with $2 \mathrm{LD}_{50}$ mouse-adapted influenza virus developed significantly (up to 16 times) higher anti-M2e antibody response in comparison to mice immunized with $\mathrm{pEx} 4 \mathrm{M} 2 \mathrm{e}$ vaccine using the same immunization protocol. This was in correlation with the increased survival rate (near to $67 \%$ vs $50 \%$ ) observed in animals immunized with pEx 4M2e-LAMP-2a DNA in comparison to mice immunized with pEx $4 \mathrm{M} 2 \mathrm{e}$.
\end{abstract}

Keywords: influenza A; matrix protein 2 ectodomain; NS1; LAMP-2a; DNA vaccine

\section{Introduction}

Influenza A viruses (IAVs) cause highly contagious seasonal acute respiratory disease, which rapidly spreads in human population. Besides the high morbidity, influenza death toll represents globally approximately 250,000500,000 deaths per year in average epidemic season. The threat of influenza epidemics or even pandemics invokes

"Corresponding author. E-mail: virufkos@savba.sk; phone: +421-2-59302450.

Abbreviations: $\mathrm{aa}$ = amino acid; $\operatorname{IAV}(\mathrm{s})$ = influenza A virus(es); LAMP-2a = lysosome-associated membrane protein 2 isoform $\mathrm{A} ; \mathrm{M} 2=$ matrix protein $; \mathrm{M} 2 \mathrm{e}=$ ectodomain of $\mathrm{M} 2 ; \mathrm{MAb}=$ monoclonal antibody; MHC II = major histocompatibility complex class II; NS1 = nonstructural protein $1 ; \mathrm{pEx}=$ expression plasmid pTriEx-4; PFU = plaque-forming unit a great concern of scientists and public health authorities. The best disease prevention remains regular vaccination with IAV strains recommended by WHO. However, these seasonal vaccines have to be updated yearly to maximize their protective potential depending on unpredictable and frequent changes in virus surface glycoproteins (WHO, 2016).

Beside the seasonal influenza vaccines containing live attenuated IAVs, inactivated virus or mix of the surface glycoproteins, hemagglutinin and neuraminidase, there is a strong effort to develop broadly cross-protective recombinant vaccines conferring hetero-subtype antiviral immunity. Such vaccine would be effectively protective for a period of several years without the need of yearly revaccination. Development of cross-protective 'universal' influenza vaccine depends on the choice of appropriate conserved influenza antigen, whose antigenic properties 
remain unchanged among various IAV strains, such as NP, HA2 gp, or ectodomain of M2 (Staneková and Varečková, 2010; Scorza et al., 2016; Nachbagauer and Krammer, 2017; Tomčíková and Varečková, 2019; Kostolanský et al., 2020). To enhance the immunogenicity of conserved parts of IAV proteins, numerous approaches have been developed utilizing functional vectors directing the immune response to induce broad T-cell or humoral immunity. Various viruses, which are able to deliver large amount of antigen for the presentation to the immune system, are exploited as vectors. These are baculoviruses, adenoviruses, vaccinia virus, but also virus-like particles. Another strategy to enhance the immunogenicity of an antigen is the combination with new adjuvants or nanoparticles. Moreover, DNA- or mRNA-based vaccines have been described (Soema et al., 2015).

The extracellular domain of M2 protein (M2e) as a perspective immunogen for the heteroprotective vaccine is a subject of numerous studies (Deng et al., 2015). Although previous studies showed that vaccination of mice with M2e provided protection against lethal challenge (Slepushkin et al., 1995; Neirynck et al., 1999; Wu et al., 2007; Ebrahimi et al., 2012), the immunogenicity of M2e was low, prompting more research to improve it. Specific anti-M2e antibodies are not virus-neutralizing, but are able to inhibit viral replication through antibody Fc-effector function by engaging Fc-recognizing receptors on immune cells by antibody-dependent cell-mediated cytotoxicity (ADCC) or interplay with complement by complement-dependent cytotoxicity (CDC) (Jagerlehner et al., 2004; Wang et al., 2008), leading to the elimination of infected cells. Moreover, specific targeting of M2e (or other conserved viral proteins) to immune system can induce specific long-term immune response mediated by antibodies as well as by effector and memory CD4 ${ }^{+} \mathrm{T}$-cells (Schotsaert et al., 2013; Eliasson et al., 2017; Jegaskanda et al., 2017; Kolpe et al., 2017).

The amino acid sequence of M2e is highly conserved among influenza A viruses (IAVs) with only minor differences among IAVs defined by evolutionary adaptation to the host organism. We designed a DNA vaccine against a wide range of IAVs based on M2e. The consensus amino acid sequence of M2e of human IAVs corresponding to the consensus sequence of IAVs of H3 phylogenetic lineage was chosen as antigen (Ito et al., 1991; Furuse et al., 2009). To increase its immunogenicity, we targeted M2e to the endolysosomal pathway using lysosome-associated membrane protein-2 isoform A (LAMP-2a), which was characterized previously (Janvier and Bonifacino; 2005; Eskelinen et al., 2005; Zhou et al., 2005; Kaushik and Cuervo, 2012). The DNA vaccine represented by expression plasmid pTriEx-4 DNA comprised the ORF for [NS1-124aa $4 \mathrm{xM} 2 \mathrm{e}$ ] or ORF for [NS1-124aa 4xM2e LAMP-2a]. The M2e intracellular localization and its co-localization with endolysosomal compartments in VERO cells was analyzed by immunofluorescence. The immunogenicity and protection efficacy of these two newly designed experimental vaccines were compared in BALB/c mice using mouseadapted IAV. The production of anti-M2e antibodies and their protective effect was quantified. The advantage of the NS1 protein truncation represents a promising approach not only for DNA vaccines, but especially in the development of new generation of live attenuated influenza vaccines (Richt and García-Sastre, 2009). With the intention to utilize such DNA vaccine as a part of live attenuated recombinant vaccine virus, we incorporated into our DNA construct truncated NS1 gene (NS1-124aa). Such truncated NS1 gene should not inhibit the interferon pathway activation after infection, thus enabling stronger immune response.

\section{Materials and Methods}

Cells. Madin-Darby canine kidney (MDCK) cells and VERO cells (cell line collection of Institute of Virology, Biomedical Research Center, SAS) were seeded and maintained in Dulbecco's modified Eagle's medium (DMEM, Lonza) supplemented with $2 \mathrm{mM}$ L-glutamine (Lonza), 1x antibiotic-antimycotic solution (Lonza) and 10\% heat-inactivated fetal bovine serum (GE Healthcare).

Viruses. The mouse-adapted recombinant influenza A/Mississippi/1/1985(xH3N2) (A/Miss) with replaced HA and NA genes into the $\mathrm{A} /$ Puerto Rico/8/1934 was used as a challenge virus in DNA vaccination experiments in BALB/c mice. The virus originated from the collection of the Institute of Virology, Biomedical Research Center, SAS. The infectious virus titer was determined by modified plaque assay using immunostaining with nucleoprotein-specific monoclonal antibody (Hollý et al., 2017). The $50 \%$ lethal dose for BALB/c mice was determined as $52 \mathrm{PFU}$ per mouse, administered intranasally in $40 \mu \mathrm{l}$ phosphate buffered saline under light isoflurane anesthesia (Kostolanský et al., 2002).

Antibodies. Mouse monoclonal antibody (MAb) against M2e (anti-M2e) was purchased from Santa Cruz Biotechnology (14C2, sc-32238) (Wise et al.,2012). The MAb was used at the concentration of $0.2 \mu \mathrm{g} \cdot \mathrm{ml}^{-1}$ and $2 \mu \mathrm{g} \cdot \mathrm{ml}^{-1}$ for western blot detection and indirect immunofluorescence analysis, respectively. Nucleoprotein-specific MAb (107L) produced in house was used as the detector antibody for immunostaining in plaque assay at the concentration 1.5 Mg/ml (Varečková et al., 1995; Hollý et al., 2017).

Laboratory animals. The six-week-old female BALB/c mice used in this study were purchased from the Faculty of Medicine, Masaryk University, Brno, Czech Republic. The animals were treated according to the approval and standards of the European Union and State Veterinary and Food Administration of the 
Slovak Republic. The State Veterinary and Food Administration of the Slovak Republic specifically approved this study (permission number 1179/16-221a). The fundamental ethical principles and animal welfare requirements were respected.

Plasmid preparation and cloning. For DNA vaccination we used pTriEx-4 expression plasmid comprising modified NS segment of IAV. Two forms of plasmid DNA vaccine were constructed: first (Fig. 1a), the NS1 truncated to first 124 aa (NS1-124) was joined in frame with four repetitions of M2e separated by Gly-Gly-Gly-Gly-Ser (G4S) sequences using a Gly-Gly-Gly-Gly (G4) linker. The ORF was terminated by three consecutive stop codons and the whole sequence was inserted to pTriEx-4 vector by ligation independent cloning (Novagen). This plasmid was designated pEx 4M2e. In the second sequence (Fig. 1b), the NS1-124 is followed in frame by 2A sequence derived from teschovirus A (TV-A) to maintain synthesis of two separate peptides from the same ORF through ribosome skipping mechanism (Kim et al., 2011). The NS1-124 2A was joined through the Gly-Gly-Gly-Gly-Gly-Gly (G6) linker to four repetitions of M2e, each separated by Ala-Ala-Ala-Pro-Gly-Ala-Ala (AAAPGAA) sequence. The C-terminal end of lysosome-associated membrane glycoprotein-2 isoform A (LAMP-2a, GenBank: J04183.1) corresponding to its transmembrane domain (TD, 11 aa) and cytoplasmic tail (CT, 25 aa) (Eskelinen et al., 2005) was added at the C-terminal end of the target sequence through G6 linker. The ORF was terminated by three consecutive stop codons and the sequence was inserted to pTriEx-4 vector by ligation independent cloning (Novagen). This plasmid was designated here as pEx 4M2e-LAMP-2a.

The ORFs of modified NS1 were amplified by PCR with Q5 High-Fidelity DNA polymerase (New England BioLabs) according to manufacturer's instructions using a pair of primers designed for cloning into pTriEx-4 vector: NS1fwd 5'-GACGAC GACAAGATGGATCCAAACACTGTGTCAAGCTTTCAG-3' and NS1rev 5'-GAGGAGAAGCCCGGTTCAAACTTCTGACCTAATT GTTCCCGCC-3', the underlined sequences are complementary to pTriEx-4 vector cloning site. Amplicons were cloned to pTriEx-4 vector using protocol supplied with the pTriEx-4 Ek/LIC Vector Kit (Novagen). Cloned sequences were verified by sequencing (Microsynth AG, Switzerland).

The pEx 4M2e plasmid was used as a template to prepare plasmid with only one copy of M2e sequence with the same backbone as pEx 4M2e. For that purpose, the inverse PCR with the pair of primers delFwd 5'-TGATAATAAGCGGCCGCAGTG-3', delRev 5'-GTCGGAGCTGTCGTTACACC-3' using Q5 High-Fidelity DNA polymerase (New England BioLabs) was employed. The reaction was subjected to agarose gel electrophoresis, the expected amplicons purified and recircularized with T4 DNA ligase (ThermoFisher Scientific). The deletion in the acquired plasmid pTriEx-4 NS1-124 1M2e, designated here as pEx 1M2e, was verified by sequencing (Microsynth AG, Switzerland).

In addition to the plasmids expressing the fusion proteins, the control expression plasmids pTriEx-4 M2 (abbr. pEx M2, carrying the whole M2 ORF) and pTriEx-4 NS1 (abbr. pEx NS1, carrying the whole NS1 ORF) were prepared as follows: Whole RNA was extracted from the allantoic fluid containing A/Puerto Rico/8/1934(H1N1) virus using NucleoSpin RNA II Purification Kit (Macherey-Nagel) according to the manufacturer's instructions. The RNA was reverse-transcribed with M-MuLV Reverse Transcriptase (Fermentas) using random hexamer primers. Acquired cDNA was subjected to PCR using Phusion HighFidelity PCR Master Mix (Finnzymes) with primers specific for the NS1 ORF (GenBank: AF389122.1; Košík et al., 2011) or for the M2 ORF (GenBank: AF389121.1). In the case of M2 ORF, the primer pair M2fwd 5'-GACGACGACAAGATGAGTCTTCTAAC CGAGGTCGAAACGTAC-3' and M2rev 5'-GAGGAGAAGCCCG GTTTACTCCAGCTCTATGCTGACAAAATGACC-3' was used. Underlined overhang sequences are necessary for cloning to pTriEx- 4 vector. The amplicons were cloned the same way as mentioned above and described previously (Košík et al., 2011). The correctness of cloning was confirmed by sequencing (Microsynth AG, Switzerland). All pTriEx-4 derived plasmids were tested for the protein expression in cell culture by indirect immunofluorescence (IF) and western blotting as described below.

Cell transfection. Cultures of MDCK or VERO cells were grown on 6 - or 24 -well plates to $70-80 \%$ confluence. Then the cell cultures were transfected with plasmid DNA (pDNA) using TurboFect in vitro Transfection Reagent (ThermoFisher Scientific) according to the manufacturer's recommendation. Transfected cells were used in further experiments.

Immunofluorescence (IF) analysis. Twenty-four hours post transfection the cells were washed with phosphate-buffered saline (PBS), fixed in $4 \%$ paraformaldehyde for $10 \mathrm{~min}$ at room temperature (RT), washed with PBS, permeabilized with $0.1 \%$ Triton X-100 for 10 min and washed again with PBS. The samples were subsequently blocked in blocking solution ( $1 \%$ bovine serum albumin fraction $\mathrm{V}, 0.5 \%$ goat normal serum, 0.05\% Tween 20, $0.2 \mathrm{M}$ glycine in PBS) for $1 \mathrm{~h}$ at RT, washed and incubated with M2e-specific MAb (14C2) for $1.5 \mathrm{~h}$ or overnight at $4^{\circ} \mathrm{C}$. Then the samples were washed three times with $0.05 \%$ Tween 20 in PBS (PBST), incubated with FITC-labeled polyclonal goat anti-mouse antibodies (Dako) for $1.5 \mathrm{~h}$ at $4^{\circ} \mathrm{C}$, washed again 3 times with PBST and mounted on microscope slides using Duolink Mounting Medium with DAPI (Olink Bioscience). The samples were observed and micrographs taken on Leica DM6000B microscope or on confocal microscope Zeiss LSM 510 META.

Western blot (WB). Twenty-four post transfection MDCK cells were scraped off in an ice-cold PBS, lysed with mammalian protein extraction reagent (M-PER, Thermo Scientific) with added protease inhibitor cocktail (Halt, Thermo Scientific) and centrifuged at $16,000 \mathrm{xg}$ for $5 \mathrm{~min}$. Subsequently the $6 \mathrm{x}$ sample buffer containing SDS (10\%) and dithiothreitol $(600 \mathrm{mM})$ was added to protein extracts (supernatants), the samples were heated at $100^{\circ} \mathrm{C}$ for $10 \mathrm{~min}$, cooled and loaded onto a 5\%/15\% SDS PAGE gel. Separated proteins were transferred to the nitrocellulose 
membrane at constant voltage $(30 \mathrm{~V})$ overnight. The membrane was blocked with $5 \%$ non-fat dried milk in Tris buffered saline (TBS) for $1 \mathrm{~h}$ and incubated with M2e-specific MAb (14C2) diluted in $1 \%$ non-fat dried milk in TBS supplemented with $0.1 \%$ Tween 20 (TBST) for $1.5 \mathrm{~h}$. Further, the membrane was washed 3 times for 10 min with TBST and incubated with polyclonal goat anti-mouse AlexaFluor 790-conjugated antibodies (Life Technologies) diluted 1:20,000 in 1\% non-fat dried milk in TBST. The membranes were washed 3 times in TBST, rinsed with distilled water and scanned on Odyssey CLx Imaging System (Li-Cor). The resulting images were analyzed with Image Studio Lite v2.1. software.

Colocalization analysis. The localization pattern of the designed peptide (4M2e LAMP-2a) within the cell compartments was evaluated by IF analysis and by colocalization with early and late endosomes using CellLight ${ }^{\circ}$ Early EndosomeRFP and CellLight Late Endosome-RFP BacMam 2.0 kits (Life technologies) according to the manufacturer's instructions. Briefly, six hours after transfection of MDCK cells with pDNA using TurboFect in vitro transfection reagent (ThermoFisher Scientific) the cells were transduced with baculoviruses expressing fluorescent fusion constructs of either Rab5a-RFP for early endosomes or Rab7a-RFP for late endosomes at the multiplicity of infection $(\mathrm{MOI})=10$. Twenty-eight hours after transfection, i.e. $22 \mathrm{~h}$ after transduction, the cells were fixed with $4 \%$ paraformaldehyde for $15 \mathrm{~min}$ at RT and IF analysis for detection of additional antigens was performed as described above. Colocalization of fluorescent signals of RFP-tagged protein and FITC-labeled antigen was evaluated with Carl Zeiss LSM Image examiner software. Colocalization of fluorescent signals was expressed as percentage of square root of Pearson's coefficient of voxel colocalization of fluorescent signals (FITC, RFP) in $z$-stack of micrographs acquired using confocal microscope. Alternatively, the colocalization of fluorescent signals was evaluated on fluorescent intensity line profile of micrograph with uniform optical slice thickness for each laser-scanned fluorescent channel.

Mice immunization and challenge. Blood samples were collected from facial vein of individual animals without anesthesia. Samples were allowed to clot and the sera were separated by centrifugation and stored at $-20^{\circ} \mathrm{C}$ until use. Groups of mice ( 4 groups, $n=18$ ) were aseptically intramuscularly injected in caudal tights (right:left, 1:1) with $100 \mu \mathrm{g}$ of pDNA diluted in $100 \mu \mathrm{l}$ of sterile PBS (50 $\mu \mathrm{l}$ per tight) with 3 doses in two-week intervals. Two weeks after the third immunization, the mice were challenged with $2 \mathrm{LD}_{50}$ of $\mathrm{A} /$ Miss virus administered intranasally in $40 \mu \mathrm{l}$ of sterile PBS under light isoflurane anesthesia. Survival of mice was monitored for two weeks. Blood samples were collected four to six hours before each immunization, virus challenge and 14 days post infection, i.e. before mice were humanely euthanized by cervical dislocation under deep anesthesia.
ELISA of anti-M2e antibodies. Enzyme-linked immunosorbent assay (ELISA) was employed to determine the level of anti-M2e antibodies in mice sera after DNA vaccination and virus challenge. Ninety-six-well high binding microplates (Greiner Bio-One) were coated overnight with $40 \mathrm{ng} /$ well of M2e peptide (GenScript) diluted in PBS at $4^{\circ} \mathrm{C}$. The amino acid sequence of M2e used in ELISA was the same as designed in DNA vaccines except the Cys residues were replaced by Ser residues. On the following day, plates were rinsed with PBS and saturated with $1 \%$ ovalbumin in PBS for $1 \mathrm{~h}$ at RT. Mouse sera from individual animals obtained after each DNA vaccination and virus challenge were twofold serially diluted in duplicates in microplates in $1 \%$ ovalbumin in PBS and sera were incubated $1.5 \mathrm{~h}$ at RT. Then plates were washed 3 times with PBST for $5 \mathrm{~min}$ and incubated with polyclonal goat antimouse HRP-conjugated antibodies (Thermo Scientific) diluted 1:5,000 in $1 \%$ ovalbumin in PBS for $1.5 \mathrm{~h}$ at RT. The plates were washed 4 times with PBST for $5 \mathrm{~min}$ and subsequently the peroxidase substrate OPD (o-phenylenediamine dihydrochloride) diluted in McIlvaine solution ( $\mathrm{pH}=5.5$ ) with $0.03 \%$ hydrogen peroxide was added. The reaction was stopped after $6 \mathrm{~min}$ of incubation by adding $2 \mathrm{M}$ sulfuric acid and the absorbance at $492 \mathrm{~nm}$ was measured on Epoch Microplate Spectrophotometer (BioTek). Antibody titer was calculated as the endpoint of average sample absorbance per duplicate of reciprocal dilution above the cut-off value, defined as two-times the average of the background, i.e. negative control absorbance. The anti-M2e MAb 14C2 (Santa Cruz Biotechnology) was included as positive control on each microplate and an irrelevant MAb was used as negative control.

Statistics. To assess mice survival, the Kaplan-Meier survival curve analysis was employed using GraphPad Prism 6.01 software. The survival curves of groups of mice were compared with log-rank (Mantel-Cox) test and the p-values and confidence intervals (CI) were noted. M2e-specific antibody titer in mouse sera determined for each time point of immunization within a group of mice and among the groups were analyzed with two-way ANOVA. Tukey's multiple comparison test was used to compare means of antibody titer among different time points of mice immunization within a group and among groups of treated mice.

\section{Results and Discussion}

Design and construction of DNA vaccines containing ectodomain of M2 protein of influenza $A$ viruses

We have prepared three DNA vaccines on the background of versatile expression vector pTriEx-4(Novagen). Simple genetic fusion of N-terminal part of NS1 protein to four copies of M2e was used to prepare pEx 4M2e plasmid (Fig. 1a). Similarly, construct carrying one copy of M2e was 
(a)

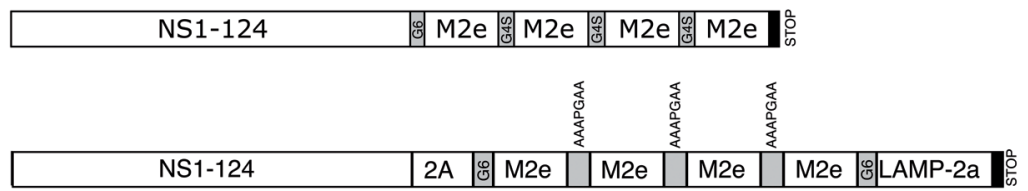

(c) number of origin of consensus sequence sequences IAVs

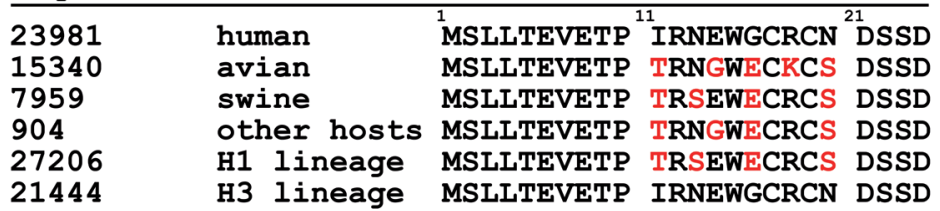

Fig. 1

Design of DNA vaccine vectors and M2e sequence analysis

Schematic of modified NS segment of IAV used for construction of DNA vaccine plasmid (a) pEx 4M2e and (b) pEx 4M2e-LAMP-2a. STOP - three subsequent stop codons, 2A - ribosome skipping sequence from teschovirus A, M2e - matrix protein 2 ectodomain, G6 - GGGGGG linker, G4S - GGGGS linker, LAMP-2a - transmembrane domain and cytoplasmic tail of lysosome-associated membrane glycoprotein-2 isoform A, (c) Consensus sequences of M2e of groups of IAVs by species origin acquired from NIAID IRD (Zhang et al., 2016) and analyzed with UGENE v1.26.1 software (Okonechnikov et al., 2012). The number of sequences used in multiple sequence alignment to acquire consensus sequence is showed. Consensus sequence of M2e of human IAV origin was used in all DNA vaccines. Differences from human origin IAV M2e consensus sequence are highlighted in red.

prepared ( $\mathrm{pEx} 1 \mathrm{M} 2 \mathrm{e})$. The third DNA vaccine (pEx 4M2eLAMP-2a, Fig. 1b) was designed to target the protein to the endo-lysosome pathway in order to enhance specific antibody response to vaccine antigen.

$D N A$ vaccine based on $p$ TriEx-4 vector expressing recombinant proteins containing $\mathrm{M} 2 \mathrm{e}$

Four copies of 23 amino acid long M2e (aa 2-24 of M2 protein) were used in two constructs. Amino acid sequence of M2e used in this study corresponds to consensus sequence of H3-lineage of human IAV strains (Fig. 1c). The initial methionine was omitted in vaccine design as it is naturally removed during protein synthesis (Lamb et al., 1985). Two constructs, namely pEx 4M2e (Fig. 1a) and pEx 1M2e, were prepared as simple fusion of N-terminal 124 amino acids of NS1 protein with four or one copies of M2e, respectively.

In $\mathrm{pEx} 4 \mathrm{M} 2 \mathrm{e}-\mathrm{LAMP}-2 \mathrm{a}$ construct (Fig. $1 \mathrm{~b}$ ), the N-terminal NS1 part was separated from the rest of the sequence by 2 A ribosome skipping sequence to facilitate the expression of two separate proteins from the same ORF (Kim et al., 2011). To target the M2e to the endo-lysosome pathway, we used the C-terminal sequence of lysosome-associated membrane glycoprotein-2 isofom A (LAMP-2a), which corresponds to its transmembrane domain (TD) and cytoplasmic tail (CT) (Eskelinen et al., 2005). The C-terminal sequence of LAMP-2a contains GYXX $\emptyset$ motif serving as lysosome sorting signal (Janvier and Bonifacino, 2005). Luminal domain of LAMP-2a, which was practically replaced with four copies of $\mathrm{M} 2 \mathrm{e}$, is by its nature heav- ily glycosylated to withstand the proteases and acidic environment of lysosomes (Eskelinen et al., 2005). Four copies of M2e were linked with AAAPGAA sequences to facilitate disordered structure targeted to endo-lysosome pathway via LAMP-2a lysosome sorting signal to mimic natural distribution of LAMP-2a protein within the cell compartments (Gough and Fambrough, 1997; Subach et al., 2009). Previously, it was shown that the LAMP-2a is engaged in chaperone-mediated autophagy (CMA) and presumably in cross-presentation of endogenous peptides on MHC class II molecules in professional antigen-presenting cells (APC) (Zhou et al., 2005; Kaushik and Cuervo, 2012). Disordered and presumably unglycosylated M2e (Zebedee et al, 1985) is expected to be rapidly degraded in lysosome lumen and, in APCs, presented on MHC class II molecules (Zhou et al., 2005; Kaushik and Cuervo, 2012). As the majority of LAMPs are suggested to traffic via the plasma membrane to lysosomes (Janvier and Bonifacino, 2005), it can result in more effective induction of specific immune response, even if the target cells are not APCs.

\section{In vitro characterization of DNA vaccines}

To examine recombinant protein expression from prepared DNA vaccines and the intracellular protein localization, we employed indirect immunofluorescence (IF) with specific anti-M2e MAb. The product of pEx 4M2e plasmid clearly accumulated in cell nuclei with weak diffuse distribution within cell cytoplasm. The same signal distribution, but of weaker intensity, was observed for pEx 1M2e plasmid (Fig. 2a). Evident nuclear accumula- 
(a)

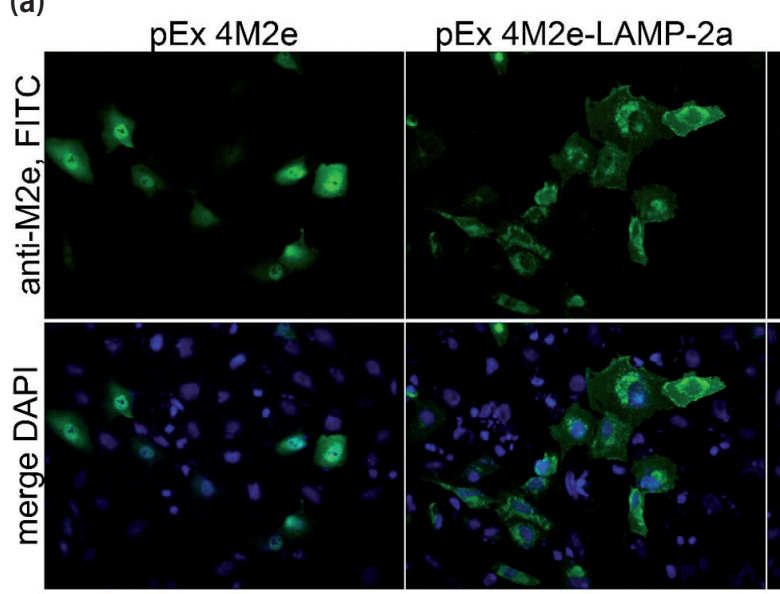

$\mathrm{pEx} M 2$

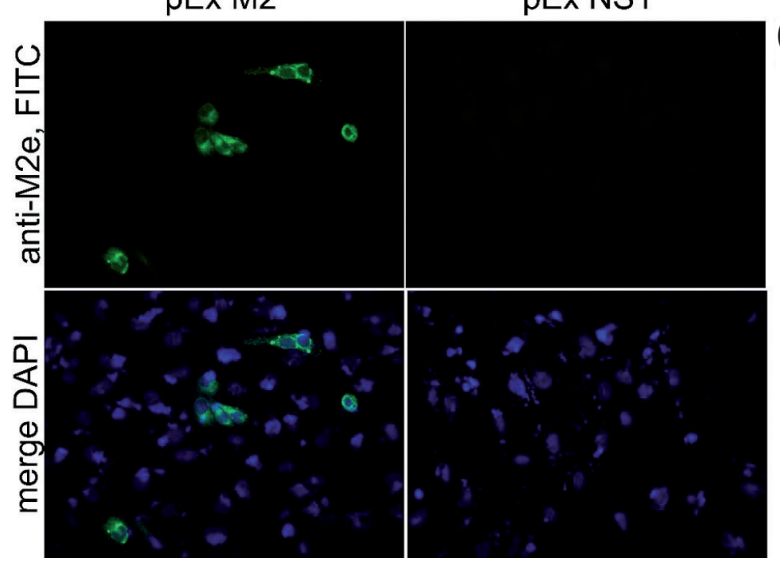

pEx $1 \mathrm{M} 2 \mathrm{e}$
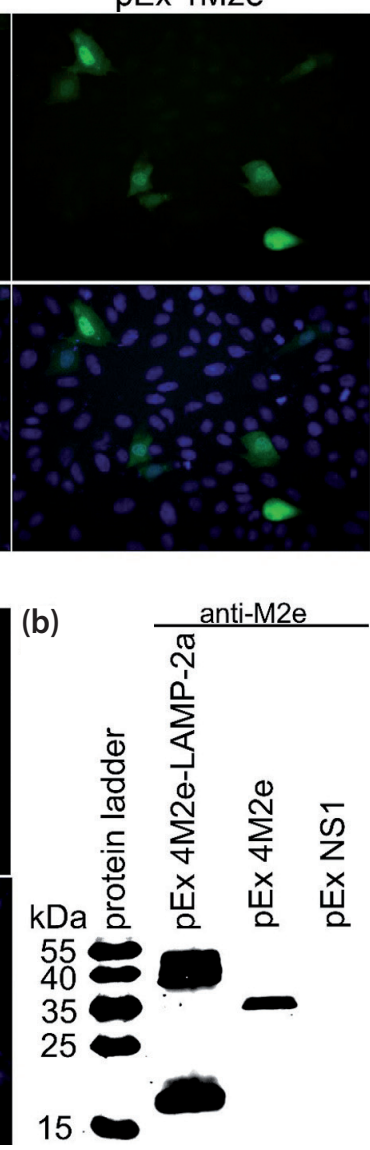

Fig. 2

Expression of recombinant proteins from prepared plasmids was evaluated by indirect immunofluorescence and western blotting

(a) Expression and intracellular localization of recombinant proteins produced from prepared plasmids was evaluated in transiently transfected VERO cells by indirect immunofluorescence using M2especific MAb (14C2) and FITC-conjugated secondary antibody (green color). Cell nuclei were stained with DAPI (blue). Plasmids pEx M2 and pEx NS1 were used as positive and negative control for M2especific MAb, respectively. (b) Western blot analysis of protein samples from transiently transfected MDCK cells. M2especific MAb (14C2) was used as detector antibody. AlexaFluor 790 was used as secondary antibody against detector $\mathrm{MAb}$ and the signal was visualized on Odyssey ${ }^{\circ} \mathrm{CLx}$ (Li-Cor).

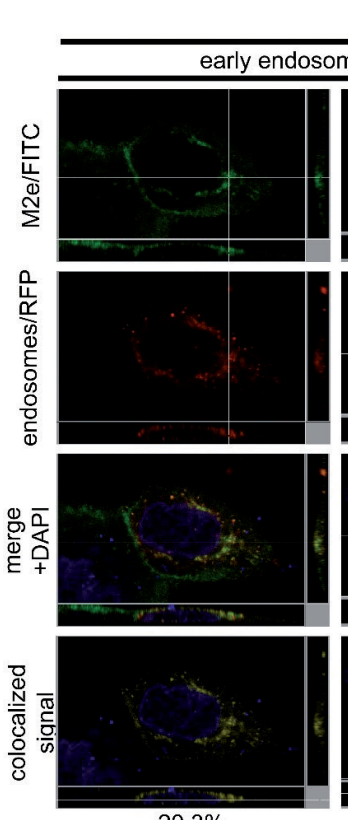

$20,3 \%$

pEx 4M2e-LAMP-2a
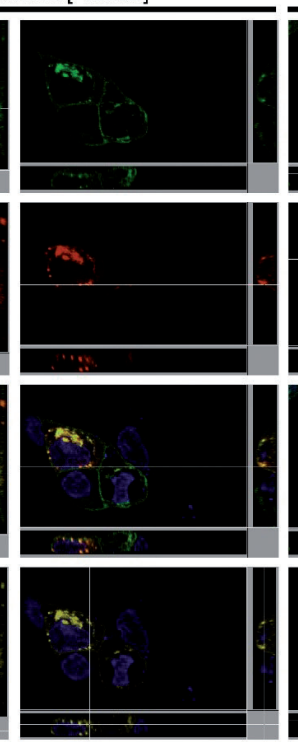

$37,2 \%$
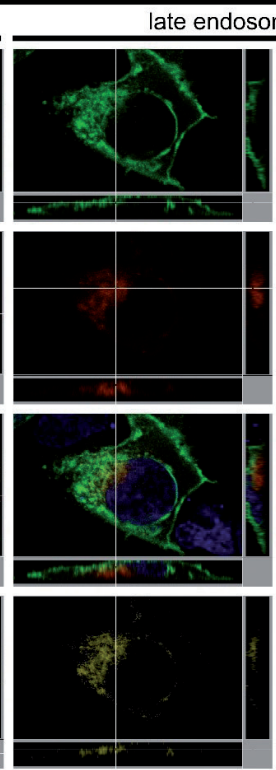

$0,2 \%$
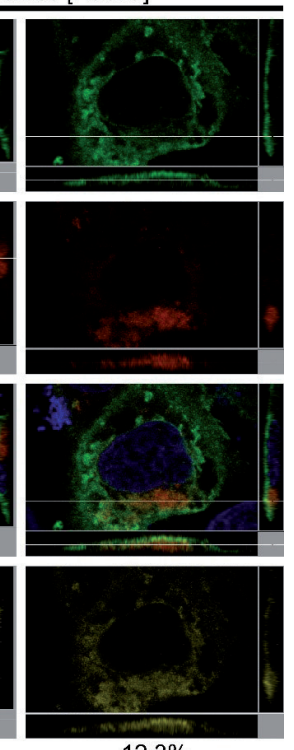

$12,3 \%$
Fig. 3

Subcellular localization was analyzed by confocal microscopy

Visible is the localization of polypeptide 4M2e-LAMP-2a in the cell membranes - strongest signal is in the nuclear membrane, cytoplasmic membrane, perinuclear region, and in the cytoplasm of distinguishable vesicles. From the co-localization of fluorescent signal for $\mathrm{M} 2 \mathrm{e}$ and early/late endosomes (RFP), respectively, it can be assumed that there is a higher co-localization between early endosomes and 4M2e-LAMP2a (4\%-37\%) than between late endosomes and this protein $(0.2 \%-12 \%)$. No signal for $4 \mathrm{M} 2 \mathrm{e}$ was observed in the cell nucleus. 
tion was most probably caused by the presence of nuclear localization signal (NLS) within the N-terminal 124 aa of NS1 protein, together with absent nuclear export signal (NES), normally present in C-terminal part of full length NS1 protein (Li et al., 2015). The expression of recombinant protein from pEx 4M2e was confirmed by western blot, showing one specific product with expected molecular weight around $35 \mathrm{kDa}$ (Fig. 2b). However, the western blot analysis of pEx 1M2e gave only a faint signal (data not shown), probably due to lower number of available epitopes recognized by the same detector MAb.

Cells transfected with pEx 4M2e-LAMP-2a showed distinct localization pattern of M2e protein in comparison to pEx 4M2e and pEx 1M2e plasmids. The fluorescence signal was strongly associated with membrane structures within cells, particularly in the perinuclear region, with vesicles under the plasma membrane, plasma membrane itself and partially with the nuclear membrane (Fig. 2a). Two specific expression products were detected in western blot analysis (Fig. 2b), with molecular weight approximately $18 \mathrm{kDa}$ and $40 \mathrm{kDa}$, most likely corresponding to peptides 4M2e-LAMP-2a and NS1-124aa-2A-4M2e-LAMP$2 a$, respectively. This is in agreement with the observation that the ribosome skipping process is not absolute (Kim et al., 2011). However, the localization of native LAMP-2a within nuclear membrane was not previously observed (Gough and Fambrough, 1997; Janvier and Bonifacino, 2005; Subach et al., 2009; Kaushik and Cuervo, 2012). We suggest that its nuclear membrane localization is due to ineffective ribosomal skipping, resulting in the expression of full-length protein with NLS in its N-terminal region together with the lysosome targeting signal at its C-terminus, which probably leads to the protein being stuck at the interface between the nucleus and the cytoplasm.

\section{Co-localization of 4M2e-LAMP-2a with endosomes}

As the immunofluorescence analysis of transfected cells showed distinct localization pattern of pEx 4M2eLAMP-2a in comparison with pEx 4M2e and pEx 1M2e (Fig. 2a), we decided to further investigate its subcellular localization by confocal microscopy. We analyzed the colocalization of $\mathrm{pEx} 4 \mathrm{M} 2 \mathrm{e}-\mathrm{LAMP}-2 \mathrm{a}$ expression product in transiently transfected cells with early (Rab5a/RFP) and late (Rab7a/RFP) endosomes. A strong co-localization signal of $\mathrm{pEx} 4 \mathrm{M} 2 \mathrm{e}-\mathrm{LAMP}-2 \mathrm{a}$ with early endosomes was observed in analyzed cells (Fig. 3 and $4 \mathrm{a}$ ). Though it varied from $4 \%$ to $37 \%$ (Fig. 3), the pEx 4 M2e-LAMP-2a signal strongly matched the early endosomes (Fig. 4a). On the other hand, the co-localization of pEx 4M2e-LAMP-2a with late endosomes of analyzed cells was weaker, within the range of $0.2 \%$ to $16 \%$ (Fig. 3). Moreover, pEx 4M2e-LAMP2 a signal did not match late endosomes as strictly as we observed in the case of early endosomes (Fig. 4a,b). Overall fluorescent signal distribution of $\mathrm{pEx} 4 \mathrm{M} 2 \mathrm{e}-\mathrm{LAMP}-2 \mathrm{a}$ was evidently bound to cell membrane structures, particularly to plasma and nuclear membranes, to the vesicles under the plasma membrane and in the perinuclear region. Colocalization of whole M2 protein expressed from control plasmid pEx M2 with early and late endosomes was similar to the co-localization of $\mathrm{pEx} 4 \mathrm{M} 2 \mathrm{e}-\mathrm{LAMP}-2 \mathrm{a}$ with late endosomes (Fig. 4b, c and d). Moreover, as expected, M2 protein, as a transmembrane protein naturally forming an ion channel, was strictly associated with cell membrane structures (Fig. 4c,d). On the contrary, protein expressed from $\mathrm{pEx} 4 \mathrm{M} 2 \mathrm{e}$ had diffuse distribution in the cytoplasm as well as in cell nuclei (Fig. 4e). The distribution pattern of the protein expressed from $\mathrm{pEx} 4 \mathrm{M} 2 \mathrm{e}-\mathrm{LAMP}-2 \mathrm{a}$ pointed to the modified M2 protein being targeted to the endolysosomal pathway. Our observation showed the protein distribution in transiently transfected and fixed cells at steady state. However, as showed by Subach et al. (2009), expression and trafficking of LAMP-2a is a dynamic process. Our results, comprising only the transmembrane domain and cytoplasmic tail of LAMP-2a as a sorting signal, are in good agreement with published data concerning native LAMP-2a distribution and its trafficking within the cells, despite not all proteins being expressed through ribosome skipping on 2A sequence as discussed above (Gough and Fambrough, 1997; Janvier and Bonifacino, 2005; Subach et al., 2009; Kaushik and Cuervo, 2012).

\section{M2e DNA vaccine immunization of $B A L B / c$ mice and its protectivity}

The immunogenicity of DNA vaccine constructs was verified in vivo. Three groups of BALB/c mice were immunized repeatedly with 3 doses of the same immunogen in 14 day-intervals and subsequently infected with lethal dose of mouse-adapted influenza A/Mississippi/1/85(H3N2) virus. The induction of M2e-specific antibody response was measured by ELISA 14 days after each immunization and 14 days after the challenge. Comparison of antibody production after immunization with $\mathrm{pEx} 1 \mathrm{M} 2 \mathrm{e}$ and $\mathrm{pEx}$ $4 \mathrm{M} 2 \mathrm{e}$ (Fig. 5a) confirmed the importance of multiple copies of M2e motif in induction of antibody response, as the presence of only one copy of M2e in the plasmid did not induce any detectable M2e-specific antibodies even after repeated administration. Statistically significant increase of anti-M2e antibodies ( $<$ 0.001) was observed in sera of A/Mississippi IAV-infected mice immunized with the plasmid pEx 4M2e-LAMP2a targeting M2e to the endolysosomes in comparison to mice non-immunized or immunized with pEx 4M2e (Fig. 5a). Our results showed that in spite of M2e being a weak immunogen, its targeting into the endolysosomes can increase the induction 


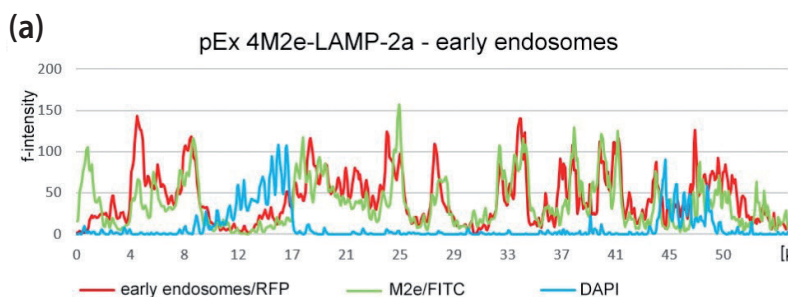

(b)

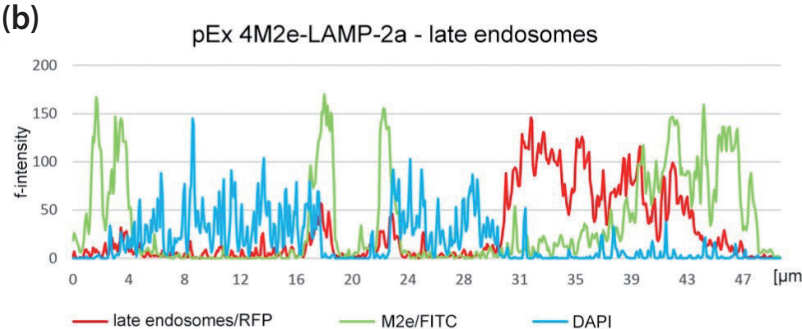

(c)

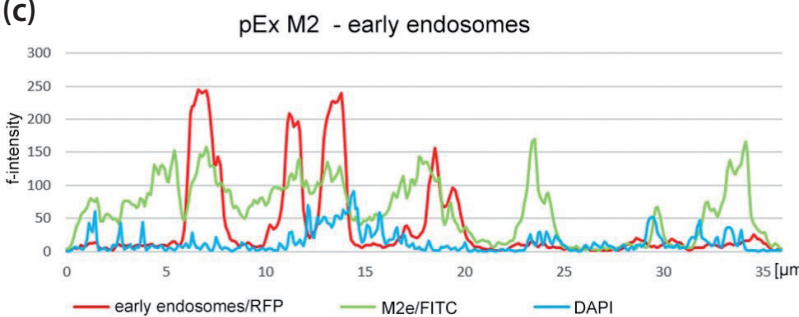

(d)

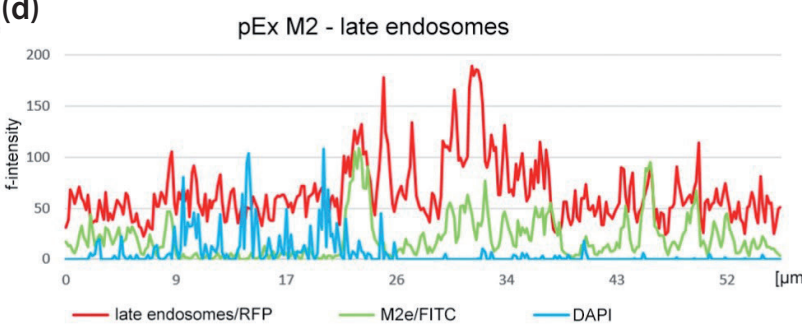

(e)

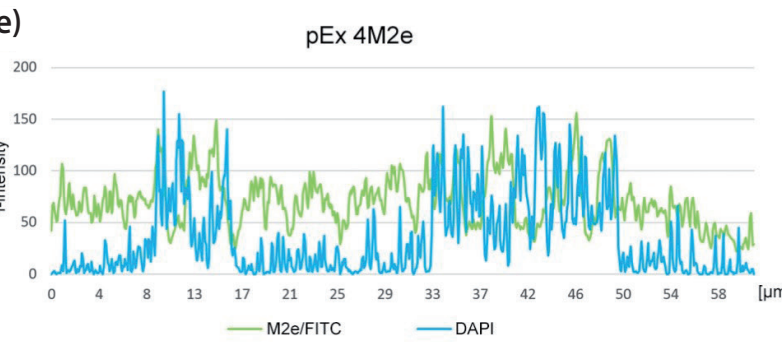

of M2e-specific antibodies conferring the improved protection to mice infected with lethal dose $\left(2 \mathrm{LD}_{50}\right)$ of IAV (Fig. 5b). The 50\% survival rate of virus-challenged mice immunized with pEx $4 \mathrm{M} 2 \mathrm{e}$ was increased up to $67 \%$ in group of mice immunized with $\mathrm{pEx} 4 \mathrm{M} 2 \mathrm{e}-\mathrm{LAMP}-2 \mathrm{a}$. With respect to the conserved nature of $\mathrm{M} 2 \mathrm{e}$, at least in human isolates, it could be supposed that the protection mediated by anti-M2e antibodies can be effective also against influenza A viruses of other subtypes.

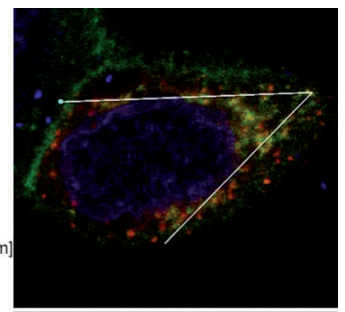

Fig. 4

Fluorescence intensity line profile analysis of micrographs

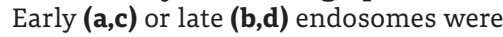
labeled with recombinant Rab5a-RFP or Rab7a-RFP protein, respectively, using CellLight ${ }^{\circ}$ BacMam 2.0 labelling kits (red). Expression of recombinant proteins from plasmid-transfected MDCK cells (a-e) was detected by indirect immunofluorescence using M2e-specific MAb and anti-mouse FITC-labeled secondary antibody (green). Cell nuclei were stained with DAPI (blue). Fluorescence signals of RFP and FITC were acquired from the same optical slice through cell plane with $0.8 \mu \mathrm{m}$ thickness or $0.6 \mu \mathrm{m}$ for DAPI. Fluorescent intensity line profiles correspond to micrographs on the right side of each profile. The start point $(0 \mu \mathrm{m})$ on profile graph corresponds to start point (white dot) of the line drawn on micrograph. Fluorescence intensity line profile of early endosomes and (a) pEx 4M2e-LAMP-2a and (c) pEx M2 are shown. Similarly, late endosomes and (b) pEx 4M2e-LAMP-2a and (d) pEx M2 are shown. Distribution of (e) pEx 4M2e expression product within cell and fluorescence intensity line profile is shown.
In summary, we showed that the immunogenicity of pTriEx-4 DNA vaccine comprising gene for truncated Nterminal 124 amino acids of NS1 protein and four copies of M2e can be increased by fusion with the C-terminal sequence of lysosome-associated membrane glycoprotein-2 isoform A (LAMP-2a). Such modification contributed significantly to the antibody response of mice to M2e, probably as a consequence of its better presentation on MHC II mediated by the presence of LAMP-2a in the DNA 
(a)

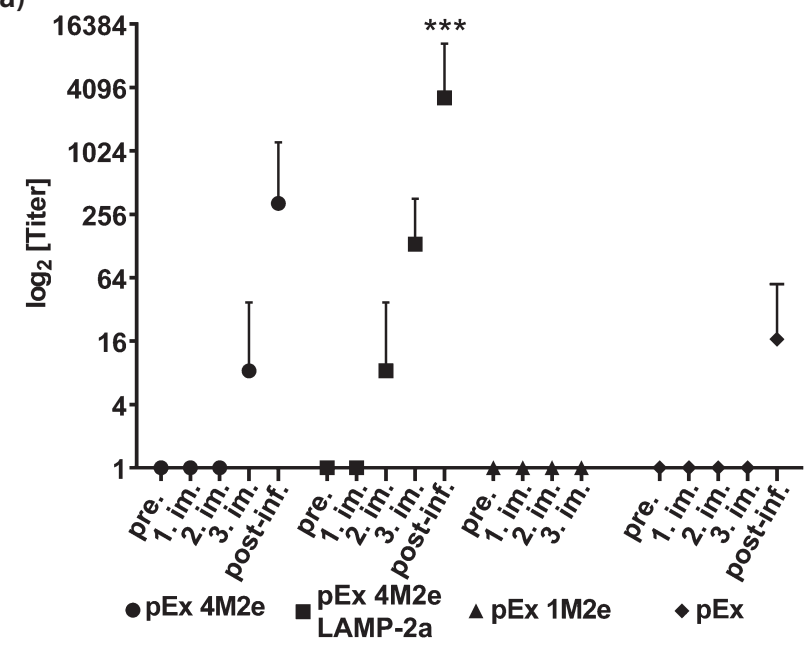

(b)

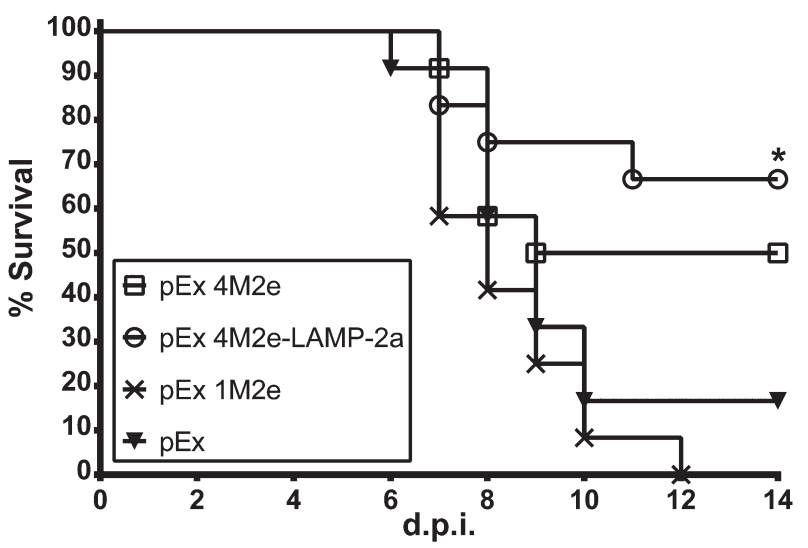

Fig. 5

Immune response and protection following $\mathrm{pTriEx} 4 \mathrm{M} 2 \mathrm{e}$ vaccination

Groups of $18 \mathrm{BALB} / \mathrm{c}$ mice (6-week-old, female) were intramuscularly immunized with $100 \mu \mathrm{\mu g}$ of plasmid DNA into both right and left flank á $50 \mu \mathrm{g}$, three times in 14 days interval. Serum samples from each animal were prepared before first immunization (N), after each immunization $(1,2,3)$ and 14 days after virus challenge. On the day 42 the mice were challenged intranasally with $2 \mathrm{LD}_{50}$ of $\mathrm{A} / \mathrm{Miss}$ influenza virus $\left(10^{4} \mathrm{PFU} / \mathrm{mouse}\right)$. On the $14^{\text {th }}$ days post infection after final blood sampling, mice were euthanized. Statistical significance of differences in titers of specific antibodies in sera was evaluated (a) (see Material and Methods). Survival of immunized mice after influenza challenge was monitored daily and recorded in Kaplan-Meier survival plot (b).

vaccine, enabling the M2e-targeting to the endo-lysosomal pathway. We suggest this approach to be considered when designing influenza vaccines with broad spectrum of antiviral efficacy.

Several research groups already developed experimental IAV-based virus-vectored vaccines employing reverse genetic method for the construction of IAV with modified NS segment carrying an antigen or a functional peptide (reviewed by Li et al., 2013). Such vaccine is able to induce specific humoral as well as cellular antiviral immune response (Sereinig et al., 2006; Tabynov et al., 2014; Jindra et al., 2015). The modification of NS1 described in our experiments could represent further improvement of such vaccines by enrichment of immune response mediated by anti-M2e protective antibodies.

Acknowledgment. Authors are thankful for the skilled technical assistance to Margita Mišovičová. This work was supported by grants from Scientific Grant Agency of the Ministry of Education of the Slovak Republic and Slovak Academy of Sciences: No 2/0106/17 (FK), 2/0048/19 (EV) and 2/0090/21 (KT), and from the Slovak Research and Development Agency grant APVV-17-0445 (EV).

\section{References}

Deng L, Cho KJ, Fiers W, Saelens X (2015): M2e-Based Universal Influenza A Vaccines. Vaccines 3, 105-136. https://doi. org/10.3390/vaccines 3010105

Ebrahimi SM, Dabaghian M, Tebianian M, Jazi MH (2012): In contrast to conventional inactivated influenza vaccines, 4xM2e.HSP70c fusion protein fully protected mice against lethal dose of $\mathrm{H} 1, \mathrm{H} 3$ and $\mathrm{H} 9$ influenza $\mathrm{A}$ isolates circulating in Iran. Virology 430, 63-72. https:// doi.org/10.1016/j.virol.2012.04.015

Eliasson DG, Omokanye A, Schon K, Wenzel UA, Bernasconi V, Bemark M, Kolpe A, El Bakkouri K, Ysenbaert T, Deng L, Fiers W, Saelens X, Lycke N (2017): M2e-tetramerspecific memory $\mathrm{CD} 4 \mathrm{~T}$ cells are broadly protective against influenza infection. Mucosal Immunol. 11, 273-289. https://doi.org/10.1038/mi.2017.14

Eskelinen E-L, Cuervo AM, Taylor MRG, Nishino I, Blum JS, Dice JF, Sandoval IV, Lippincott-Schwartz J, August JT, Saftig P (2005): Unifying nomenclature for the isoforms of the lysosomal membrane protein LAMP2. Traffic 6, 1058-1061. https://doi.org/10.1111/j.16000854.2005.00337.x

Furuse Y, Suzuki A, Kamigaki T, Oshitani H (2009): Evolution of the $\mathrm{M}$ gene of the influenza $\mathrm{A}$ virus in different host species: large-scale sequence analysis. Virol. J. 6, p.13. https://doi.org/10.1186/1743-422X-6-67

Gough NR, Fambrough DM (1997): Different steady state subcellular distributions of the three splice variants of 
lysosome-associated membrane protein LAMP-2 are determined largely by the $\mathrm{COOH}$-terminal amino acid residue. J. Cell Biol. 137, 1161-1169. https://doi. org/10.1083/jcb.137.5.1161

Hollý J, Fogelová M, Jakubcová L, Tomčíková K, Vozárová M, Varečková E, Kostolanský F (2017): Comparison of infectious influenza A virus quantification methods employing immune-staining. J. Virol. Methods 247, 107-113. https://doi.org/10.1016/i.jviromet.2017.06.004

Ito T, Gorman OT, Kawaoka Y, Bean WJ, Webster RG (1991): Evolutionary analysis of the influenza $A$ virus $M$ gene with comparison of the M1 and M2 proteins. J. Virol. 65, 5491-5498. https://doi.org/10.1128/JVI.65.10.5491$\underline{5498.1991}$

Jagerlehner A, Schmitz N, Storni T, Bachmann M (2004): Influenza A VaccineBased on the Extracellular Domain of M2: Weak Protection Mediated via Antibody-Dependent NK Cell Activity. J. Immunol. 172, 5598-5605. https:// doi.org/10.4049/jimmunol.172.9.5598

Janvier K, Bonifacino JS (2005): Role of the endocytic machinery in the sorting of lysosome-associated membrane proteins. Mol. Biol. Cell 16, 4231-4242. https://doi. org/10.1091/mbc.e05-03-0213

Jegaskanda S, Co MDT, Cruz J, Subbarao K, Ennis FA, Terajima M (2017): Induction of H7N9-Cross-Reactive AntibodyDependent Cellular Cytotoxicity Antibodies by Human Seasonal Influenza A Viruses that are Directed Toward the Nucleoprotein. J. Infect. Dis. 215, 818-823. https://doi.org/10.1093/infdis/jiw629

Jindra C, Huber B, Shafti-Keramat S, Wolschek M, Ferko B, Muster T, Brandt S, Kirnbauer R (2015): Attenuated recombinant influenza A virus expressing HPV16 E6 and E7 as a novel therapeutic vaccine approach. PLoS One 10, e0138722. https://doi.org/10.1371/journal.pone.0138722

Kaushik S, Cuervo AM (2012): Chaperone-mediated autophagy: a unique way to enter the lysosome world. Trends Cell. Biol. 22, 407-417. https://doi.org/10.1016/j. tcb.2012.05.006

Kim JH, Lee SR, Li LH, Park HJ, Lee KY, Kim MK, Shin BA, Choi SY (2011): High cleavage efficiency of a 2A peptide derived from porcine teschovirus- 1 in human cell lines, zebrafish and mice. PLoS One 6, e18556. https://doi. org/10.1371/journal.pone.0018556

Kolpe A, Schepens B, Fiers W, Saelens X (2017): M2-based influenza vaccines: recent advances and clinical potential. Expert. Rev. Vaccines 16, 123-136. https://doi.org/10.10 80/14760584.2017.1240041

Kostolanský F, Mucha V, Slováková R, Varečková E (2002): Natural influenza A virus infection of mice elicits strong antibody response to HA2 glycopeptide. Acta Virol. 46, 229-236.

Kostolanský F, Tomčíková K, Briestenská K, Mikušová M, Varečková E (2020): Universal anti-influenza vaccines based on viral HA2 and M2e antigens. Acta Virol. 64, 417-426. https://doi.org/10.4149/av 2020 408

Košík I, Krejnusová I, Bystrická M, Poláková K, Russ G (2011): $\mathrm{N}$-terminal region of the $\mathrm{PB} 1-\mathrm{F} 2$ protein is responsible for increased expression of influenza A viral protein
PB1. Acta Virol. 55, 45-53. https://doi.org/10.4149/ av_2011_01_45

Lamb RA, Zebedee SL, Richardson CD (1985): Influenza virus M2 protein is an integral membrane protein expressed on the infected-cell surface. Cell 40, 627-633. https://doi. org/10.1016/0092-8674(85)90211-9

Li J, Arévalo MT, Zeng M (2013): Engineering influenza viral vectors. Bioengineered 4, 9-14. https://doi.org/10.4161/ bioe. 21950

Li J, Yu M, Zheng W, Liu W (2015): Nucleocytoplasmic shuttling of influenza A virus proteins. Viruses 7, 2668-2682. https://doi.org/10.3390/v7052668

Nachbagauer R, Krammer F (2017): Universal influenza virus vaccines and therapeutic antibodies. Clin. Microbiol. Infect. 23, 222-228. https://doi.org/10.1016/i. cmi.2017.02.009

Neirynck S, Deroo T, Saelens X, Vanlandschoot P, Jou, WM, Fiers $W$ (1999): A universal influenza A vaccine based on the extracellular domain of the M2 protein. Nat. Med. 5, 1157-1163. https://doi.org/10.1038/13484

Okonechnikov K, Golosova O, Fursov M, UGENE Team (2012): Unipro UGENE: a unified bioinformatics toolkit. Bioinformatics 28, 1166-1167. https://doi.org/10.1093/ bioinformatics/bts091

Richt JA, García-Sastre A(2009): Attenuated influenza virus vaccines with modified NS1 proteins. Curr. Top. Microbiol. Immunol. 333, 177-195. https://doi.org/10.1007/978-3540-92165-3_9

Sereinig S, Stukova M, Zabolotnyh N, Ferko B, Kittel C, Romanova J, Vinogradova T, Katinger H, Kiselev O, Egorov A (2006): Influenza virus NS vectors expressing the mycobacterium tuberculosis ESAT- 6 protein induce $\mathrm{CD} 4+$ Th1 immune response and protect animals against tuberculosis challenge. Clin. Vaccine Immunol. 13, 898-904. https://doi.org/10.1128/CVI.00056-06

Schotsaert M, Ysenbaert T, Neyt K, Ibanez LI, Bogaert P, Schepens B, Lambrecht BN, Fiers W, Saelens X (2013): Natural and long-lasting cellular immune responses against influenza in the M2e-immune host. Mucosal Immunol. 6, 276-287. https://doi.org/10.1038/mi.2012.69

Scorza FB, Tsvetnitsky V, Donnelly JJ (2016): Universal influenza vaccines: Shifting to better vaccines. Vaccine $34,2926-$ 2933. https://doi.org/10.1016/j.vaccine.2016.03.085

Slepushkin VA, Katz JM, Black RA, Gamble WC, Rota PA, Cox NJ (1995): Protection of mice against influenza A virus challenge by vaccination with baculovirusexpressed M2 protein. Vaccine 13, 1399-402. https:// doi.org/10.1016/0264-410X(95)92777-Y

Soema PC, Kompier R, Amorij JP, Kersten GF (2015): Current and next generation influenza vaccines: Formulation and production strategies. Eur. J. Pharm. Biopharm. 94, 251-263. https://doi.org/10.1016/j.ejpb.2015.05.023

Staneková Z, Varečková E (2010) Conserved epitopes of influenza $A$ virus inducing protective immunity and their prospects for universal vaccine development. Virol. J. 7, 351. https://doi.org/10.1186/1743-422X-7-351

Subach FV, Subach OM, Gundorov IS, Morozova KS, Piatkevich KD, Cuervo AM, Verkhusha VV (2009): Monomeric 
fluorescent timers that change color from blue to red report on cellular trafficking. Nat. Chem. Biol. 5, 118-126. https://doi.org/10.1038/nchembio.138

Tabynov K, Kydyrbayev Z, Ryskeldinova S, Yespembetov B, Zinina N, Assanzhanova N, Kozhamkulov Y, Inkarbekov D, Gotskina T, Sansyzbay A (2014): Novel influenza virus vectors expressing Brucella L7/L12 or Omp16 proteins in cattle induced a strong $\mathrm{T}$-cell immune response, as well as high protectivness against B. abortus infection. Vaccine 32, 2034-2041. https://doi.org/10.1016/j. vaccine.2014.02.058

Tomčíková K, Varečková E (2019): Different mechanisms of the protection against influenza A infection mediated by broadly reactive HA2-specific antibodies. Acta Virol. 63, 347-365. https://doi.org/10.4149/av 2019408

Varečková E, Betáková T, Mucha V, Soláriková L, Kostolanský F, Waris M, Russ G (1995): Preparation of monoclonal antibodies for the diagnosis of influenza A infection using different immunization protocols. J. Immunol. Methods 180, 107-116. https://doi.org/10.1016/00221759(94)00307-I

Wang R, Song A, Levin J, Dennis D, Zhang NJ, Yoshida H, Koriazova L, Madura L, Shapiro L, Matsumoto A, Yoshida H, Mikayama T, Kubo RT, Sarawar S, Cheroutre H, Kato S (2008): Therapeutic potential of a fully human monoclonal antibody against influenza A virus M2 protein. Antiviral Res. 80, 168-177. https://doi.org/10.1016/j. antiviral.2008.06.002

Wise HM, Hutchinson EC, Jagger BW, Stuart AD, Kang ZH, Robb N, Schwartzman LM, Kash JC, Fodor E, Firth AE, Gog JR, Taubenberger JK, Digard P (2012): Identification of a Novel Splice Variant Form of the Influenza A Virus M2 Ion Channel with an Antigenically Distinct Ectodomain. PLoS Pathog. 8, e1002998. https://doi. org/10.1371/journal.ppat.1002998

World Health Organization, Geneva, 2016, S. Fact Sheet 211: Influenza, http://www.who.int/mediacentre/factsheets/ fs211/en/

Wu F, Huang JH, Yuan XY, Huang WS, Chen YH (2007): Characterization of immunity induced by M2e of influenza virus. Vaccine 25, 8868-8873. https://doi.org/10.1016/i. vaccine.2007.09.056

Zebedee SL, Richardson CD, Lamb RA (1985): Characterization of the influenza virus M2 integral membrane protein and expression at the infected-cell surface from cloned cDNA. J. Virol. 56, 502-511. https://doi.org/10.1128/ JVI.56.2.502-511.1985

Zhang Y, Aevermann BD, Anderson TK, Burke DF, Dauphin G, Gu Z, He S, Kumar S, Larsen CN, Lee AJ, Li X, Macken C, Mahaffey C, Pickett BE, Reardon B, Smith T, Stewart L, Suloway C, Sun G, Tong L, Vincent AL, Walters B, Zaremba S, Zhao H, Zhou L, Zmasek C, Klem EB, Scheuermann BH (2016): Influenza Research Database: An integrated bioinformatics resource for influenza virus research. Nucleic Acids Res. 45(D1), D466-D474. https://doi.org/10.1093/nar/gkw857

Zhou D, Li P, Lin Y, Lott JM, Hislop AD, Canaday DH, Brutkiewicz RR, Blum JS (2005): Lamp-2a facilitates MHC class II presentation of cytoplasmic antigens. Immunity 22, 571-581. https://doi.org/10.1016/i.immuni.2005.03.009 